\section{Distúrbios menstruais entre mulheres de 15-54 anos de idade em Pelotas, Rio Grande do Sul, Brasil: estudo de base populacional}

\author{
Menstrual disorders among women 15 to 54 years \\ of age in Pelotas, Rio Grande do Sul State, Brazil: \\ a population-based study
}

\author{
Trastornos menstruales en mujeres entre 15-54 \\ años de Pelotas, Rio Grande do Sul, Brasil: \\ estudio de base poblacional
}

Raquel Siqueira Barcelos 1 Roberta de Vargas Zanini 1 Iná da Silva dos Santos 1

\section{Resumo}

Para avaliar a prevalência de distúrbios menstruais e sua distribuição conforme paridade e características demográficas, sociais e comportamentais, entre 15-54 anos de idade, realizou-se estudo transversal de base populacional, em Pelotas, Rio Grande do Sul, Brasil, com $1.078 \mathrm{mu}$ lheres. Foram investigados sangramento menstrual excessivo, sangramento intermenstrual, ciclo menstrual curto, ciclo menstrual longo e menstruação prolongada. Foram calculadas as taxas de prevalência, com intervalos de 95\% de confiança. A prevalência global de distúrbios menstruais foi 46,4\% (43,4-49,4). O mais prevalente foi sangramento menstrual excessivo (23,2\%; 20,5-25,8), seguido de ciclo menstrual curto $(19,2 \% ; 16,6-21,8)$, ciclo menstrual longo $(14,8 \% ; 12,7-16,9)$, sangramento intermenstrual (11,1\%; 9,1-13,2) e menstruação prolongada (7,9\%; 6,3-9,4). Os distúrbios menstruais são frequentes na população, estando associados à idade maior que 34 anos, escolaridade menor que cinco anos, maior paridade, laqueadura tubária e tabagismo.

Distúrbios Menstruais; Menstruação; Saúde da Mulher 


\section{Introdução}

Os trabalhos pioneiros de Treloar et al. ${ }^{1}$ mostraram que o ciclo menstrual de cada mulher varia muito pouco ao longo da vida reprodutiva. Já os estudos que investigaram populações femininas diferentes encontraram acentuada variação geográfica quanto à idade da menarca 2,3,4,5 e menopausa 2,5,6,7 e quanto à duração da menstruação ${ }^{8}$, enquanto, para a variação regional da duração do ciclo menstrual, as evidências são mais fracas 9. Além disso, estudos mostram que, para a mesma mulher, os primeiros ciclos após a menarca e aqueles próximos à menopausa costumam apresentar maior variação na periodicidade, quantidade e duração, em comparação ao restante dos ciclos 10. A maioria dos estudos disponíveis foi realizada entre mulheres europeias, usuárias de serviços de saúde, havendo pouca informação sobre a variação do ciclo menstrual e da menstruação obtida em estudos de base populacional, o que limita ainda mais o conhecimento sobre sua variação normal.

Tanto para o uso em pesquisa quanto na área clínica, o ciclo menstrual é definido como o período entre o primeiro dia de uma menstruação e a véspera do início da menstruação seguinte 11 . Os estudos sobre o padrão da menstruação costumam considerar três características do ciclo menstrual: periodicidade, intensidade e duração. A periodicidade corresponde ao intervalo de tempo entre os sangramentos (geralmente descrito como em torno de 24-35 dias) 12; a intensidade equivale à quantidade de sangue eliminada durante a menstruação (geralmente considerada como de 30-80ml); e a duração é o número de dias em que se dão as perdas sanguíneas (geralmente variando de dois a sete dias) 13 .

A melhora no estado nutricional 14 (que antecipou a idade da menarca) 3,15, a diminuição planejada do número de gestações 16,17 e a inserção no mercado de trabalho (reduzindo o tempo de amamentação pós-parto) 18,19 levaram as mulheres contemporâneas a experimentar mais ciclos menstruais do que as mulheres do passado, facilitando o estudo da variação das características do ciclo menstrual. Assim, o objetivo do presente estudo foi estimar a prevalência de distúrbios menstruais e sua distribuição de acordo com a paridade e características demográficas, sociais e comportamentais, entre mulheres de 15-54 anos de idade, residentes em Pelotas, Rio Grande do Sul, Brasil.

\section{Metodologia}

Um estudo transversal de base populacional, parte do Consórcio de Pesquisa dos mestrandos do Programa de Pós-graduação em Epidemiologia da Universidade Federal de Pelotas (UFPel) 20, foi realizado de fevereiro a junho de 2012, para avaliar a saúde de adolescentes, adultos e idosos, moradores da zona urbana de Pelotas, cidade localizada na Região Sul do Brasil, com 328.275 habitantes. Para o atual estudo, a população alvo eram mulheres de 15-54 anos, sendo excluídas as institucionalizadas ou com alguma incapacidade mental que as impedisse de responder ao questionário.

O tamanho de amostra necessário para investigar a prevalência de distúrbios menstruais foi calculado com base nos seguintes parâmetros e estimativas: prevalência de distúrbio menstrual de $35 \% 21$, erro aceitável de três pontos percentuais, nível de 95\% de confiança, efeito de delineamento (DEF) de 2,0 e um acréscimo de $10 \%$ para perdas e recusas. Dessa forma, seria necessária uma amostra de 2.134 mulheres.

O processo de amostragem foi realizado em múltiplos estágios. Primeiramente, foram selecionados os conglomerados, utilizando dados do Censo Demográfico de 2010, fornecidos pelo Instituto Brasileiro de Geografia e Estatística (IBGE; http://www.ibge.gov.br/cidadesat/ topwindow.htm?1, acessado em 24/Jun/2011). Os 495 setores censitários da cidade foram ordenados por sua numeração, sendo essa estratégia baseada na localização geográfica. Os setores são numerados em formato de espiral, do centro para a periferia, em sentido horário. Com isso, a participação de diversos bairros da cidade, com diferentes níveis socioeconômicos, foi garantida na amostra. De acordo com o IBGE, em 2010, havia em Pelotas aproximadamente 1,4 mulher na faixa etária de interesse, por domicílio. Portanto, seria necessário visitar 1.524 domicílios para alcançar o número estimado para a amostra, sendo selecionados sistematicamente 130 setores e, em cada setor, visitados cerca de doze domicílios.

No trabalho de campo, foram visitados 1.555 domicílios (necessários para atender aos tamanhos de amostra de todos os mestrandos), nos quais foram localizadas 1.495 mulheres de 15-54 anos de idade. Tal achado evidenciou uma redução do número de mulheres nessa faixa etária de 1,4 , como estimado com base no censo de 2010 , para 0,9 por domicílio.

O questionário utilizado para coleta de dados foi pré-testado em um estudo piloto e apresentava sete perguntas com opções fechadas de resposta: 
1. Desde [seis meses atrás] o ano passado, a senhora menstruou pelo menos uma vez? ( $\mathrm{sim} /$ não);

2. A senhora está grávida no momento ou esteve grávida nos últimos 6 meses? (sim, está grávida/ sim, esteve grávida/não);

3. Desde [seis meses atrás] o ano passado, quanto a senhora perdeu de sangue na maioria das menstruações? (muita quantidade/pouca quantidade/normal);

4. Desde [seis meses atrás] o ano passado, a senhora teve pelo menos uma menstruação que desceu em menos de 3 semanas? (sim/não);

5. Desde [seis meses atrás] o ano passado, a senhora teve pelo menos uma menstruação que levou mais de 5 semanas para descer? ( $\operatorname{sim} /$ não); 6. Desde [seis meses atrás] o ano passado, a senhora teve algum sangramento entre as menstruações? (sim/não);

7. Desde [seis meses atrás] o ano passado, a senhora teve pelo menos uma menstruação que durou mais de 10 dias? (sim/não).

Pelas duas primeiras perguntas foram identificadas as mulheres com amenorreia de pelo menos seis meses, as atuais gestantes e as que estiveram grávidas em algum período nos seis meses anteriores à pesquisa, as quais foram excluídas das análises. Os desfechos investigados foram: (a) distúrbio da intensidade (sangramento menstrual excessivo); (b) distúrbios da periodicidade (ciclo menstrual curto, ciclo menstrual longo e sangramento intermenstrual); e (c) distúrbio da duração (menstruação prolongada).

Os distúrbios menstruais foram definidos da seguinte maneira:

- Sangramento excessivo: mulheres que classificaram sua perda sanguínea como em "grande quantidade", na maioria das vezes, nos seis meses anteriores à pesquisa.

- Sangramento intermenstrual: aquelas que mencionaram perda sanguínea entre uma menstruação e outra, pelo menos uma vez, nos seis meses anteriores à entrevista.

- Ciclo menstrual curto: aquelas que, nos seis meses anteriores à pesquisa, tiveram pelo menos uma vez um intervalo de tempo inferior a três semanas entre uma menstruação e outra.

- Ciclo menstrual longo: aquelas mulheres que, nos seis meses anteriores à entrevista, pelo menos uma vez, tiveram intervalo de tempo superior a cinco semanas entre uma menstruação e outra.

- Menstruação prolongada: aquelas que relataram que menstruaram por dez dias ou mais, pelo menos uma vez, nos seis meses anteriores à pesquisa.

Foram coletadas informações sobre as seguintes variáveis independentes: idade (anos completos), cor da pele (observada pela entrevistadora e classificada como branca e não branca), escolaridade (anos completos de estudo, posteriormente categorizada em 0-4, 5-8, 9-11 e 12 ou mais anos), classe econômica (classificação da ABEP; http://www.abep.org/novo/Content. aspx?ContentID=301, acessado em 27/Jun/2011), uso de método anticoncepcional (utilização de algum método anticoncepcional nos seis meses anteriores à pesquisa), paridade (número de partos), índice de massa corporal (classificado como $<25,0,25,0-29,9$ e $\geq 30,0$ ), tabagismo (sendo considerada "fumante" a mulher que fumava um ou mais cigarros por dia, há mais de um mês) e intensidade do hábito de fumar (número de cigarros fumados por dia). Para as mulheres que relataram o uso de mais de um método anticoncepcional nos seis meses anteriores à entrevista, considerou-se somente o primeiro mencionado, independentemente da cronologia e do tempo de uso.

A classificação da ABEP estima o poder de compra das pessoas e famílias empregando um sistema de pontos baseado em uma lista de posse de nove bens (automóvel, televisão em cores, rádio, empregada mensalista, máquina de lavar, vídeocassete e/ou DVD, geladeira e freezer) e na escolaridade do chefe da família. Com base nessa pontuação, as pessoas são classificadas em cinco classes econômicas (A, B, C, D e E), sendo a classe A aquela com maior poder e o E com menor poder de compra. Como havia um número pequeno de mulheres nas classes extremas, para a análise, a variável foi reagrupada em três categorias (A/B, C e D/E). Ainda para a análise, a variável "uso de método anticoncepcional" foi agrupada, e as mulheres que relataram o uso de métodos de barreira (146 reportaram o uso de preservativo pelos parceiros masculinos) e métodos naturais (cinco relataram usar tabela) foram classificadas na mesma categoria daquelas que relataram não ter usado nenhum método, uma vez que esses métodos não têm efeitos colaterais, aos quais se possa atribuir alterações menstruais 22. Para levar em conta aspectos fisiológicos na gênese dos distúrbios menstruais, a partir da idade foi construída a variável "período reprodutivo", categorizada em: adolescência (15-19 anos), menacma (20-45 anos) e climatério ( $>45$ anos).

Os dados foram coletados com o auxílio de netbooks, com uso do programa Pendragon Forms 6 (Pendragon Software Corp., Buffalo Grove, Estados Unidos), os quais eram semanalmente sincronizados a um computador de uso comum entre os mestrandos. Em seguida, o banco de dados foi transferido para o programa Stata versão 12.0 (Stata Corp., College Station, Estados Unidos), onde foi realizada a avaliação da consistên- 
cia dos dados e as análises estatísticas. Todos os dados foram coletados por entrevistadoras previamente treinadas e padronizadas pelos supervisores da pesquisa.

A prevalência global de distúrbio menstrual, no período de seis meses, foi a primeira a ser calculada. Foram consideradas no numerador as mulheres que apresentaram pelo menos um dos seis distúrbios menstruais investigados, nos seis meses anteriores à entrevista. No denominador, constaram todas as mulheres que referiram ter menstruado nos seis meses anteriores à entrevista. Posteriormente, foram calculadas as prevalências para os tipos específicos de distúrbios. Foi avaliada a distribuição de cada distúrbio de acordo com características demográficas (idade e cor da pele) e sociais (nível econômico e escolaridade), bem como conforme a paridade e variáveis comportamentais das mulheres (uso de método anticoncepcional, tabagismo e intensidade do hábito de fumar), sendo obtidos intervalos de 95\% de confiança (IC95\%) para cada estimativa. O desenho amostral foi considerado em todas as análises, de forma a corrigir as estimativas de variabilidade para a correlação intraconglomerado. Foram realizados testes qui-quadrado de heterogeneidade e tendência linear, considerando um nível de 95\% de significância. As razões de prevalências (RP) brutas e ajustadas e seus IC95\% foram calculadas por regressão de Poisson, sendo mantidas no modelo ajustado as variáveis que se associaram com cada um dos desfechos com $\mathrm{p}<0,20$ e retiradas as demais de forma retrógrada.

Este estudo foi aprovado pelo Comitê de Ética em Pesquisa da Faculdade de Medicina da UFPel. Todas as entrevistadas assinaram o Termo de Consentimento Livre e Esclarecido concordando em participar do estudo.

\section{Resultados}

Dentre as 1.495 mulheres de 15-54 anos de idade elegíveis para o estudo, 134 (9\%) não foram localizadas após três tentativas em dias e horários diferentes ou se recusaram a participar do estudo, restando 1.361 que foram entrevistadas. Dessas, 1.078 haviam menstruado pelo menos uma vez nos seis meses anteriores à pesquisa e não estavam nem estiveram grávidas no período, sendo incluídas na presente análise.

O controle de qualidade foi realizado com 179 mulheres (16,6\% da amostra), quando foi repetida a pergunta sobre paridade ("número de partos até o momento da entrevista”). O resultado obtido no teste kappa, para medir a repetição da questão, foi 0,9 , sendo considerado “excelente". O efeito de delineamento amostral para sangramento menstrual excessivo, sangramento intermenstrual, ciclo menstrual curto, ciclo menstrual longo e menstruação prolongada foi, respectivamente, 1,$1 ; 1,1 ; 1,2 ; 1,0 ;$ e 1,0.

A média de idade das mulheres entrevistadas foi 31,1 anos (DP $\pm 10,6)$. Na Tabela 1, é apresentada a distribuição da amostra. Observa-se que foi composta predominantemente por mulheres brancas (82,3\%), com idade entre $15 \mathrm{e}$ 35 anos (61\%). Quanto ao período reprodutivo da vida, $70 \%$ das mulheres encontravam-se na menacma.

Mais de um terço tinha 9-11 anos de escolaridade $(37,2 \%)$ e $47 \%$ pertenciam à classe econômica C. Mais de um terço era nulípara $(37,2 \%)$, e cerca da metade $(47,7 \%)$ tinha sobrepeso ou obesidade e era usuária de pílula como método anticoncepcional (49,9\%). Mais de dois terços nunca haviam fumado $(69,6 \%)$. Entre as fumantes, $34,4 \%$ fumavam mais de 20 cigarros/dia.

A prevalência global de distúrbios menstruais, nos seis meses anteriores ao estudo, foi $46,4 \%$ (IC95\%: 43,3-49,4). Na Figura 1, são apresentadas as frequências acumuladas de distúrbios menstruais. Mais de um quarto das mulheres (26,9\%) relatou a presença de um único distúrbio menstrual nos últimos seis meses, enquanto $19,5 \%$ referiram a presença de dois a cinco distúrbios. Dentre as mulheres com dois distúrbios menstruais, a combinação mais frequente foi de ciclo menstrual curto e ciclo menstrual longo; e houve aquelas com três - sangramento menstrual excessivo, ciclo menstrual curto e sangramento intermenstrual.

Na Tabela 2, é apresentada a prevalência de cada distúrbio menstrual de acordo com a paridade, índice de massa corporal (IMC) e características demográficas, sociais e comportamentais das mulheres. O distúrbio mais referido foi sangramento menstrual excessivo (23,2\%; IC95\%: $20,5-25,8 \%)$ e o menos prevalente foi menstruação prolongada (7,9; IC95\%: 6,3-9,4\%).

O sangramento excessivo foi mais comum entre mulheres de 35-44 anos, com escolaridade entre 0-4 anos, que tiveram quatro ou mais partos, com IMC $\geq 30,0$, que relataram ligadura tubária e fumo. $\mathrm{O}$ ciclo menstrual curto foi o segundo distúrbio mais referido (19,2\%; IC95\%: $16,6-21,8 \%)$, sendo mais frequente entre mulheres de 35-44 anos, com menos de nove anos de escolaridade, com história de quatro partos ou mais e usuárias de anticoncepcional hormonal injetável. A prevalência de ciclo menstrual longo foi $14,8 \%$ (IC95\%: 12,7-16,9\%), sendo mais frequente entre mulheres climatéricas (de 45-54 anos de idade), com IMC $\geq 30,0$ e usuárias de dispositivo intrauterino (DIU). 
Tabela 1

Distribuição da amostra de mulheres de 15-54 anos de idade, de acordo com paridade, índice de massa corporal (IMC), características demográficas, socioeconômicas, biológicas e comportamentais. Pelotas, Rio Grande do Sul, Brasil, $2012(\mathrm{~N}=1.078$ *).

\begin{tabular}{|c|c|c|}
\hline Características & $\mathrm{n}$ & $\%$ \\
\hline \multicolumn{3}{|l|}{ Faixa etária (anos) } \\
\hline $15-24$ & 372 & 34,5 \\
\hline $25-34$ & 286 & 26,5 \\
\hline $35-44$ & 271 & 25,1 \\
\hline $45-54$ & 149 & 13,8 \\
\hline \multicolumn{3}{|l|}{ Período reprodutivo } \\
\hline Adolescência & 202 & 18,6 \\
\hline Menacma & 759 & 70,0 \\
\hline Climatério & 124 & 11,4 \\
\hline \multicolumn{3}{|l|}{ Cor da pele } \\
\hline Branca & 887 & 82,3 \\
\hline Não branca & 191 & 17,7 \\
\hline \multicolumn{3}{|c|}{ Classe econômica (ABEP) } \\
\hline $\mathrm{A} / \mathrm{B}$ & 464 & 43,5 \\
\hline C & 502 & 47,0 \\
\hline $\mathrm{D} / \mathrm{E}$ & 102 & 9,6 \\
\hline \multicolumn{3}{|c|}{ Escolaridade (anos de estudo) } \\
\hline $0-4$ & 85 & 7,9 \\
\hline $5-8$ & 262 & 24,3 \\
\hline $9-11$ & 401 & 37,2 \\
\hline$\geq 12$ & 330 & 30,6 \\
\hline \multicolumn{3}{|l|}{ Paridade } \\
\hline 0 & 356 & 37,2 \\
\hline 1 & 231 & 24,1 \\
\hline 2 & 198 & 20,7 \\
\hline 3 & 102 & 10,7 \\
\hline$\geq 4$ & 71 & 7,4 \\
\hline \multicolumn{3}{|l|}{ IMC (kg/m²) } \\
\hline$<25,0$ & 521 & 52,3 \\
\hline $25,0-29,9$ & 302 & 30,3 \\
\hline$\geq 30,0$ & 173 & 17,4 \\
\hline \multicolumn{3}{|c|}{ Método anticoncepcional } \\
\hline Nenhum & 357 & 35,2 \\
\hline Pílula & 505 & 49,9 \\
\hline Hormonal injetável & 29 & 2,9 \\
\hline DIU & 26 & 2,6 \\
\hline Ligadura tubária & 96 & 9,5 \\
\hline \multicolumn{3}{|l|}{ Tabagismo } \\
\hline Não & 750 & 69,6 \\
\hline Sim & 192 & 17,8 \\
\hline Ex-fumante & 136 & 12,6 \\
\hline \multicolumn{3}{|l|}{ Cigarros/dia } \\
\hline $1-4$ & 75 & 23,1 \\
\hline $5-10$ & 106 & 32,5 \\
\hline $11-20$ & 33 & 10,1 \\
\hline$>20$ & 112 & 34,4 \\
\hline
\end{tabular}

ABEP: Associação Brasileira de Empresas de Pesquisa; DIU: dispositivo intrauterino.

* O valor máximo de informações perdidas foi de 11,1\% (120) para a variável paridade. 
Frequência acumulada de distúrbios menstruais entre mulheres de 15-54 anos de idade. Pelotas, Rio Grande do Sul, Brasil, $2012(N=1.078)$

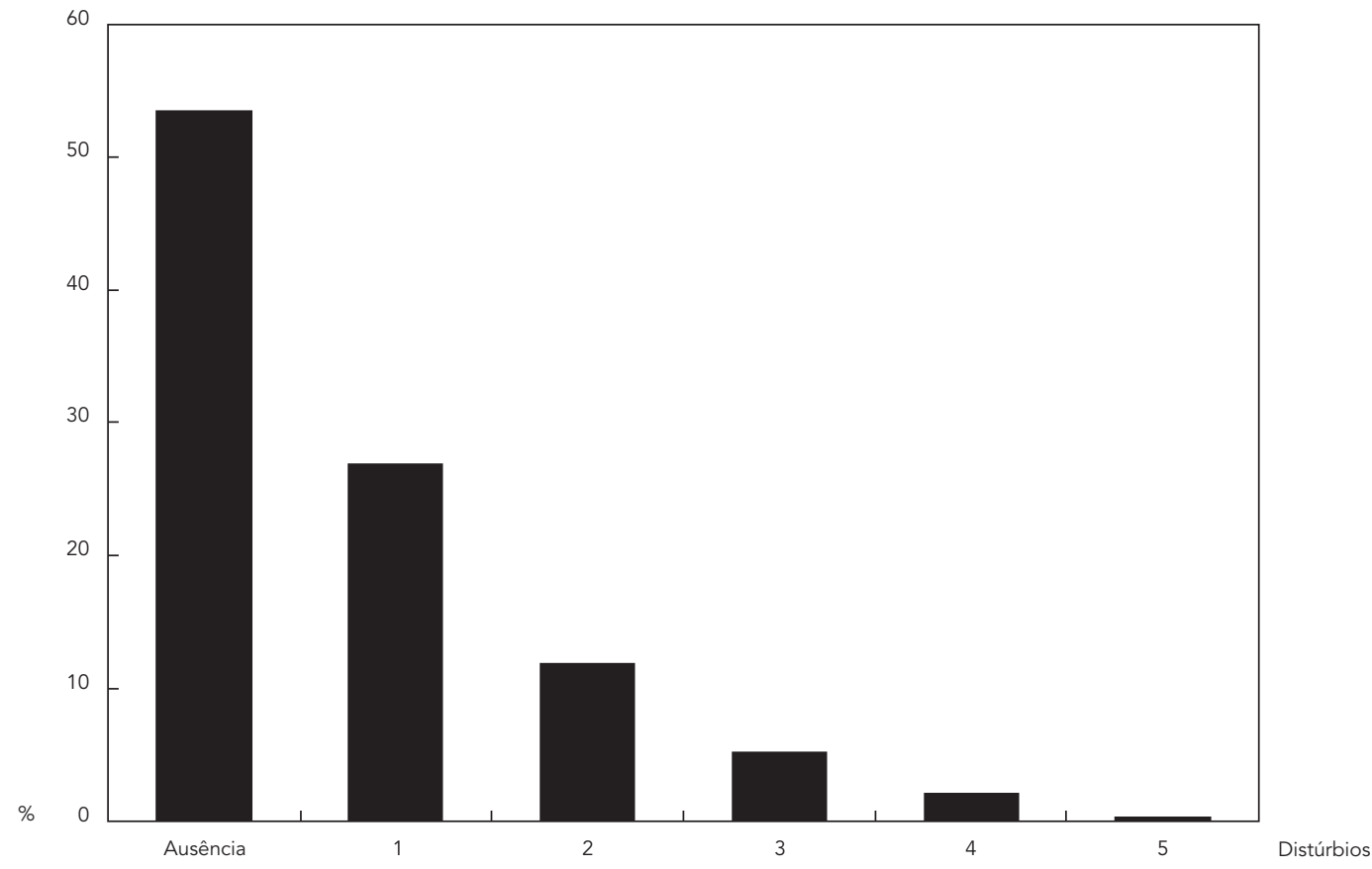

O sangramento intermenstrual apresentou prevalência de 11,1\% (IC95\%: 9,1-13,2\%) e esteve associado somente ao método anticoncepcional, sendo mais frequente entre mulheres com ligadura tubária. A prevalência de menstruação prolongada foi 7,9\% (IC95\%: 6,3-9,4\%) e ocorreu mais frequentemente entre aquelas pertencentes às classes econômicas $\mathrm{D} / \mathrm{E}$, com cinco a oito anos de escolaridade, com quatro partos ou mais, usuárias de anticoncepcional hormonal injetável e fumantes.

Nas Tabelas 3 e 4, são apresentadas as análises bruta e ajustada entre os cinco desfechos investigados e as variáveis independentes estudadas. Na análise bruta, todas as variáveis, exceto cor da pele e classe econômica, apresentaram associação estatisticamente significativa com sangramento menstrual excessivo. $\mathrm{Na}$ análise ajustada, o sangramento excessivo aumentou com a paridade e com o IMC, sendo duas vezes mais frequente entre as multíparas em comparação às nulíparas (RP = 2,2; IC95\%: 1,4-3,5) e 30\% mais frequente entre as obesas do que entre as com IMC < 25,0kg/m² (RP = 1,3; IC95\%: 1,0-1,8). Mulheres com ligadura tubária tiveram $20 \%$ mais relato de sangramento excessivo e as usuárias de pílula, $50 \%$ a menos do que as que não usavam nenhum método anticoncepcional.

$\mathrm{O}$ sangramento intermenstrual associou-se somente à variável método anticoncepcional, tanto na análise bruta (Tabela 3) quanto na ajustada (Tabela 4). Em comparação às que não usavam nenhum método anticoncepcional, a probabilidade de relatar sangramento intermenstrual foi $80 \%$ maior entre usuárias de pílula ou de hormônio injetável e duas vezes maior entre as com ligadura tubária.

O ciclo menstrual curto, que na análise bruta associou-se à idade, escolaridade, paridade e método anticoncepcional, após ajuste foi mais prevalente apenas entre as com um ( $\mathrm{RP}=1,6 ; \mathrm{IC} 95 \%$ : $1,0-2,6)$ ou com quatro ou mais partos $(\mathrm{RP}=1,8$; IC95\%: 1,0-3,3) e que usavam hormônio injetável (RP = 1,4; IC95\%: 0,9-2,3). Ressalte-se, no entanto, que para ambas as variáveis, os intervalos de confiança contêm a unidade.

As variáveis escolaridade, IMC e método anticoncepcional associaram-se com ciclo menstrual longo (Tabela 3). Na análise ajustada, o relato foi duas vezes e $20 \%$ mais frequente, 
Prevalência de distúrbios menstruais entre mulheres de 15-54 anos de idade, de acordo com paridade, índice de massa corporal (IMC), características demográficas, socioeconômicas, biológicas e comportamentais. Pelotas, Rio Grande do Sul, Brasil, 2012 (N = 1.078 *).

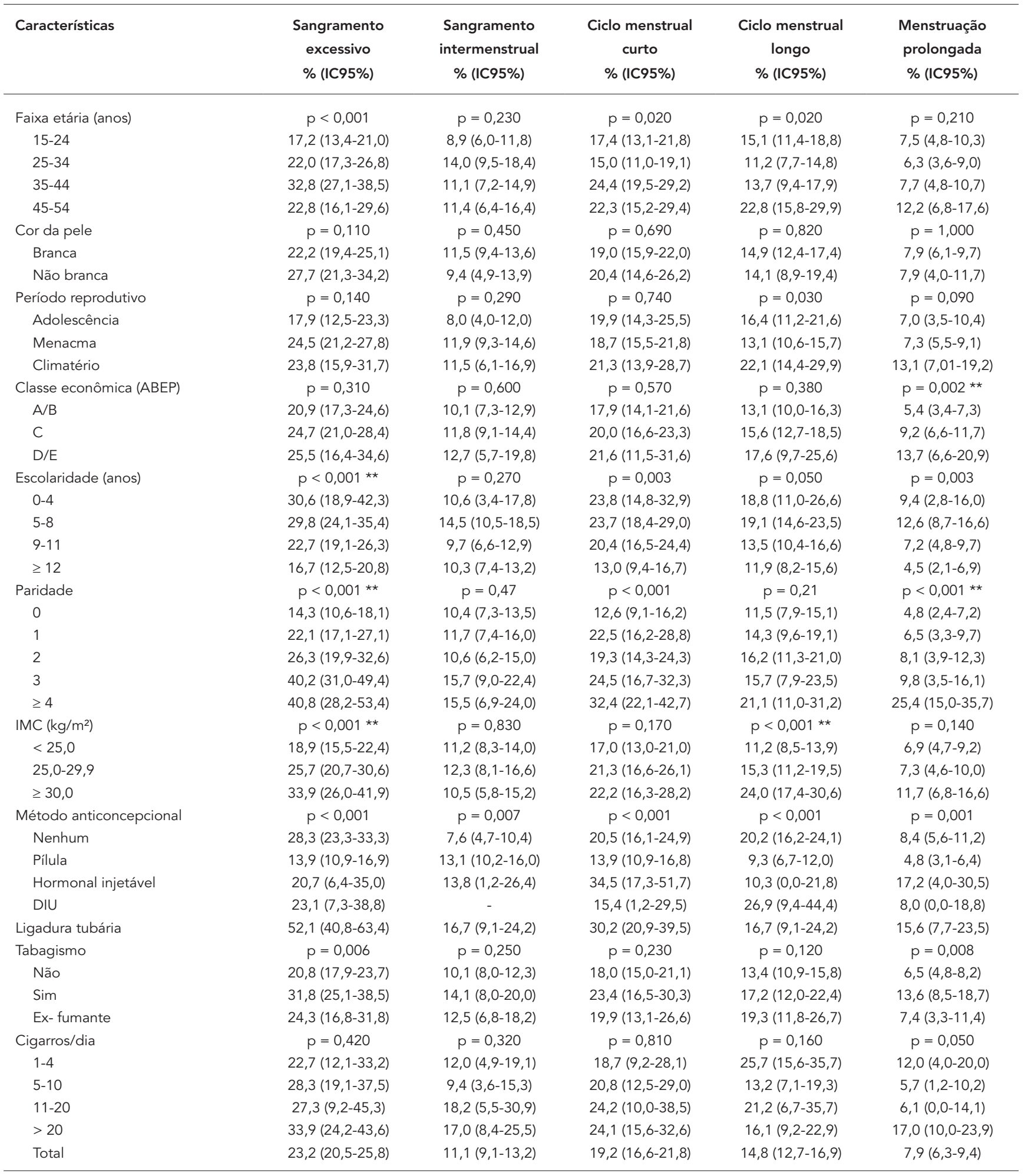

ABEP: Associação Brasileira de Empresas de Pesquisa; DIU: dispositivo intrauterino; IC95\%: intervalo de $95 \%$ de confiança ajustado para efeito de delineamento amostral.

* O valor máximo de informações perdidas foi de 11,1\% (120) para a variável paridade;

** Teste qui-quadrado de tendência linear. 
Análise bruta da associação entre distúrbios menstruais e variáveis independentes. Pelotas, Rio Grande do Sul, Brasil, 2012 (N = 1.078).

\begin{tabular}{|c|c|c|c|c|c|c|}
\hline Variável & $\mathrm{n}$ & $\begin{array}{c}\text { Sangramento } \\
\text { excessivo } \\
\text { RP (IC95\%) }\end{array}$ & $\begin{array}{c}\text { Sangramento } \\
\text { intermenstrual } \\
\text { RP (IC95\%) }\end{array}$ & $\begin{array}{c}\text { Ciclo menstrual } \\
\text { curto } \\
\text { RP (IC95\%) }\end{array}$ & $\begin{array}{c}\text { Ciclo menstrual } \\
\text { longo } \\
\text { RP (IC95\%) }\end{array}$ & $\begin{array}{c}\text { Menstruação } \\
\text { prolongada } \\
\text { RP (IC95\%) }\end{array}$ \\
\hline Idade (anos) & & $p=0,0001$ & $p=0,300$ & $p=0,009$ & $p=0,100$ * & $p=0,200$ * \\
\hline $15-24$ & 372 & 1,0 & 1,0 & 1,0 & 1,0 & 1,0 \\
\hline $25-34$ & 286 & $1,3(0,9-1,7)$ & $1,6(1,0-2,5)$ & $0,9(0,6-1,2)$ & $0,7(0,5-1,1)$ & $0,8(0,5-1,5)$ \\
\hline $35-44$ & 271 & $1,9(1,4-2,5)$ & $1,2(0,8-1,9)$ & $1,4(1,0-1,9)$ & $0,9(0,6-1,4)$ & $1,0(0,6-1,8)$ \\
\hline $45-54$ & 149 & $1,3(0,9-2,0)$ & $1,3(0,7-2,2)$ & $1,3(0,9-1,9)$ & $1,5(1,0-2,3)$ & $1,6(0,9-2,9)$ \\
\hline Cor da pele & & $p=0,090$ & $p=0,400$ & $p=0,700$ & $p=0,800$ & $p=1,000$ \\
\hline Branca & 887 & 1,0 & 1,0 & 1,0 & 1,0 & 1,0 \\
\hline Não branca & 191 & $1,2(1,0-1,6)$ & $0,8(0,5-1,3)$ & $1,1(0,8-1,5)$ & $0,9(0,6-1,5)$ & $1,0(0,6-1,8)$ \\
\hline Classe econômica (ABEP) & & $p=0,100$ * & $p=0,400$ * & $p=0,300$ * & $p=0,200$ * & $p=0,003$ * \\
\hline$A / B$ & 464 & 1,0 & 1,0 & 1,0 & 1,0 & 1,0 \\
\hline C & 502 & $1,2(0,9-1,5)$ & $1,2(0,8-1,7)$ & $1,1(0,9-1,4)$ & $1,2(0,9-1,6)$ & $1,7(1,1-2,7)$ \\
\hline $\mathrm{D} / \mathrm{E}$ & 102 & $1,2(0,8-1,8)$ & $1,3(0,7-2,3)$ & $1,2(0,7-2,0)$ & $1,3(0,8-2,2)$ & $2,5(1,3-5,0)$ \\
\hline Escolaridade (anos de estudo) & & $p=0,0001 *$ & $p=0,2$ & $p=0,0003$ * & $p=0,01$ * & $p=0,006$ \\
\hline $0-4$ & 85 & $1,8(1,2-2,9)$ & $1,0(0,5-2,0)$ & $1,8(1,1-2,9)$ & $1,6(0,9-2,7)$ & $2,1(0,8-5,1)$ \\
\hline $5-8$ & 262 & $1,8(1,3-2,4)$ & $1,4(1,0-2,0)$ & $1,8(1,3-2,6)$ & $1,6(1,1-2,4)$ & $2,8(1,5-5,1)$ \\
\hline $9-11$ & 401 & $1,4(1,0-1,8)$ & $0,9(0,6-1,4)$ & $1,6(1,1-2,2)$ & $1,1(0,8-1,7)$ & $1,6(0,8-3,1)$ \\
\hline$\geq 12$ & 330 & 1,0 & 1,0 & 1,0 & 1,0 & 1,0 \\
\hline Paridade & & $p<0,0001$ * & $p=0,400$ & $p=0,0001$ & $p=0,050$ * & $p<0,0001$ * \\
\hline 0 & 356 & 1,0 & 1,0 & 1,0 & 1,0 & 1,0 \\
\hline 1 & 231 & $1,5(1,1-2,2)$ & $1,1(0,7-1,8)$ & $1,8(1,2-2,6)$ & $1,2(0,8-1,9)$ & $1,4(0,7-2,7)$ \\
\hline 2 & 198 & $1,8(1,3-2,6)$ & $1,0(0,6-1,7)$ & $1,5(1,0-2,2)$ & $1,4(0,9-2,2)$ & $1,7(0,8-3,7)$ \\
\hline 3 & 102 & $2,8(2,0-4,0)$ & $1,5(0,9-2,6)$ & $1,9(1,3-2,9)$ & $1,4(0,7-2,5)$ & $2,1(0,9-4,9)$ \\
\hline$\geq 4$ & 71 & $2,9(1,9-4,3)$ & $1,5(0,8-2,7)$ & $2,6(1,7-3,9)$ & $1,8(1,0-3,3)$ & $5,3(2,8-10,2)$ \\
\hline IMC $\left(\mathrm{kg} / \mathrm{m}^{2}\right)$ & & $p=0,0002^{*}$ & $p=0,800$ & $p=0,100$ * & $p=0,0002$ * & $p=0,09 t$ \\
\hline$<25,0$ & 521 & 1,0 & 1,0 & 1,0 & 1,0 & 1,0 \\
\hline $25,0-29,9$ & 302 & $1,4(1,0-1,8)$ & $1,1(0,7-1,7)$ & $1,3(0,9-1,7)$ & $1,4(0,9-2,0)$ & $1,1(0,6-1,7)$ \\
\hline$\geq 30,0$ & 173 & $1,8(1,3-2,4)$ & $0,9(0,6-1,6)$ & $1,3(0,9-1,9)$ & $2,1(1,5-3,1)$ & $1,7(1,0-2,9)$ \\
\hline Método anticoncepcional & & $p<0,0001$ & $p<0,0001$ & $p=0,0002$ & $p=0,0005$ & $p=0,001$ \\
\hline Nenhum & 357 & 1,0 & 1,0 & 1,0 & 1,0 & 1,0 \\
\hline Pílula & 505 & $0,5(0,4-0,7)$ & $1,7(1,1-2,6)$ & $0,7(0,5-0,9)$ & $0,5(0,3-0,7)$ & $0,6(0,4-0,9)$ \\
\hline Hormonal injetável & 29 & $0,7(0,4-1,5)$ & $1,8(0,7-4,7)$ & $1,7(1,0-2,8)$ & $0,5(0,2-1,6)$ & $2,1(0,8-5,0)$ \\
\hline DIU & 26 & $0,8(0,4-1,7)$ & - & $0,8(0,3-1,9)$ & $1,3(0,7-2,7)$ & $0,9(0,2-3,8)$ \\
\hline Ligadura tubária & 96 & $1,8(1,4-2,4)$ & $2,2(1,3-3,8)$ & $1,5(1,0-2,2)$ & $0,8(0,5-1,3)$ & $1,9(1,0-3,4)$ \\
\hline Tabagismo & & $p=0,005$ & $p=0,400$ & $p=0,300$ & $p=0,200$ & $p=0,006$ \\
\hline Não & 750 & 1,0 & 1,0 & 1,0 & 1,0 & 1,0 \\
\hline Sim & 196 & $1,5(1,2-2,0)$ & $1,4(0,8-2,3)$ & $1,3(0,9-1,8)$ & $1,3(0,9-1,8)$ & $2,1(1,3-3,2)$ \\
\hline Ex-fumante & 136 & $1,2(0,8-1,6)$ & $1,2(0,8-2,0)$ & $1,1(0,8-1,6)$ & $1,4(0,9-2,2)$ & $1,1(0,6-2,2)$ \\
\hline
\end{tabular}

ABEP: Associação Brasileira de Empresas de Pesquisa; DIU: dispositivo intrauterino; IC95\%: intervalo de 95\% de confiança; IMC: índice de massa corporal; RP: razão de prevalência.

* Teste Wald para tendência linear.

respectivamente, entre as obesas e as usuárias de DIU, em comparação às categorias de referência.

A menstruação prolongada foi 2,3 vezes mais frequente entre as mulheres das classes econômicas $\mathrm{D} / \mathrm{E}$, em comparação às das classes $\mathrm{A} / \mathrm{B}$ (Tabela 4). Mulheres com quatro ou mais partos apresentaram probabilidade 3,5 vezes maior de relatar menstruação prolongada.

Para explorar os efeitos dos métodos anticoncepcionais, análises adicionais foram realizadas após dicotomizar a variável "método anticoncepcional" em "uso de pílula, injeção ou DIU" e 
Análise ajustada da associação entre distúrbios menstruais e variáveis independentes. Pelotas, Rio Grande do Sul, Brasil, 2012 (N =1.078).

\begin{tabular}{|c|c|c|c|c|c|c|}
\hline Variável & $\mathrm{n}$ & $\begin{array}{c}\text { Sangramento } \\
\text { excessivo } \\
\text { RP (IC95\%) }\end{array}$ & $\begin{array}{c}\text { Sangramento } \\
\text { intermenstrual } \\
\text { RP (IC95\%) }\end{array}$ & $\begin{array}{c}\text { Ciclo menstrual } \\
\text { curto } \\
\text { RP (IC95\%) }\end{array}$ & $\begin{array}{c}\text { Ciclo menstrual } \\
\text { longo } \\
\text { RP (IC95\%) }\end{array}$ & $\begin{array}{c}\text { Menstruação } \\
\text { prolongada } \\
\text { RP (IC95\%) }\end{array}$ \\
\hline Idade (anos) & & $p=0,090$ & $p=0,200$ & $p=0,200$ & $p=0,700$ * & $p=0,800$ * \\
\hline $15-24$ & 372 & 1,0 & 1,0 & 1,0 & 1,0 & 1,0 \\
\hline $25-34$ & 286 & $0,9(0,6-1,3)$ & $1,6(1,0-2,7)$ & $0,8(0,5-1,3)$ & $0,7(0,5-1,2)$ & $0,6(0,3-1,2)$ \\
\hline $35-44$ & 271 & $0,9(0,7-1,4)$ & $1,3(0,8-2,1)$ & $1,0(0,7-1,6)$ & $0,7(0,4-1,1)$ & $0,5(0,2-1,1)$ \\
\hline $45-54$ & 149 & $0,6(0,4-1,0)$ & $1,5(0,9-2,7)$ & $0,9(0,5-1,5)$ & $1,2(0,7-1,8)$ & $0,8(0,3-2,0)$ \\
\hline Cor da pele & & $p=0,900$ & $p=0,400$ & $p=0,900$ & $p=0,050$ & $p=0,200$ \\
\hline Branca & 887 & 1,0 & 1,0 & 1,0 & 1,0 & 1,0 \\
\hline Não branca & 191 & $1,0(0,7-1,4)$ & $0,8(0,5-1,3)$ & $1,0(0,7-1,4)$ & $0,6(0,3-1,0)$ & $0,6(0,3-1,2)$ \\
\hline Classe econômica (ABEP) & & $p=0,800$ & $p=0,500$ * & $p=0,700$ & $p=0,700$ * & $p=0,009$ * \\
\hline$A / B$ & 464 & 1,0 & 1,0 & 1,0 & 1,0 & 1,0 \\
\hline C & 502 & $1,1(0,8-1,3)$ & $1,1(0,7-1,5)$ & $0,9(0,7-1,3)$ & $1,0(0,7-1,4)$ & $1,6(0,9-2,6)$ \\
\hline $\mathrm{D} / \mathrm{E}$ & 102 & $0,9(0,6-1,4)$ & $1,3(0,7-2,4)$ & $1,0(0,6-1,6)$ & $1,2(0,7-2,0)$ & $2,3(1,1-4,6)$ \\
\hline Escolaridade (anos) & & $p=0,800$ & $p=0,100$ & $p=0,100$ & $p=0,100$ & $p=0,400$ \\
\hline $0-4$ & 85 & $0,9(0,6-1,6)$ & $1,0(0,4-2,4)$ & $1,2(0,7-2,0)$ & $1,1(0,6-2,2)$ & $0,7(0,3-1,9)$ \\
\hline $5-8$ & 262 & $1,2(0,8-1,7)$ & $1,5(1,1-2,2)$ & $1,4(0,9-2,0)$ & $1,5(1,0-2,1)$ & $1,4(0,7-2,8)$ \\
\hline $9-11$ & 401 & $1,1(0,8-1,6)$ & $1,1(0,7-1,7)$ & $1,3(0,9-1,8)$ & $1,0(0,7-1,5)$ & $1,1(0,5-2,4)$ \\
\hline$\geq 12$ & 330 & 1,0 & 1,0 & 1,0 & 1,0 & 1,0 \\
\hline Paridade & & $p<0,0001$ * & $p=0,600$ & $p=0,030$ * & $p=0,600$ & $p<0,0001$ * \\
\hline 0 & 356 & 1,0 & 1,0 & 1,0 & 1,0 & 1,0 \\
\hline 1 & 231 & $1,4(1,0-2,1)$ & $0,9(0,5-1,6)$ & $1,6(1,0-2,6)$ & $0,9(0,5-1,7)$ & $1,2(0,6-2,5)$ \\
\hline 2 & 198 & $1,6(1,1-2,4)$ & $0,8(0,5-1,5)$ & $1,3(0,8-2,0)$ & $0,9(0,5-1,6)$ & $1,3(0,6-3,0)$ \\
\hline 3 & 102 & $2,1(1,4-3,3)$ & $1,1(0,5-2,3)$ & $1,4(0,8-2,6)$ & $0,8(0,4-1,8)$ & $1,6(0,6-3,8)$ \\
\hline$\geq 4$ & 71 & $2,2(1,4-3,5)$ & $1,3(0,6-3,0)$ & $1,8(1,0-3,3)$ & $0,9(0,4-2,0)$ & $3,5(1,7-7,2)$ \\
\hline $\mathrm{IMC}\left(\mathrm{kg} / \mathrm{m}^{2}\right)$ & & $p=0,040 *$ & $p=0,600$ & $p=0,500$ * & $p<0,0001$ * & $p=0,700$ * \\
\hline$<25,0$ & 521 & 1,0 & 1,0 & 1,0 & 1,0 & 1,0 \\
\hline $25,0-29,9$ & 302 & $1,1(0,8-1,4)$ & $1,1(0,7-1,7)$ & $1,1(0,8-1,6)$ & $1,3(0,9-1,9)$ & $0,7(0,4-1,2)$ \\
\hline$\geq 30,0$ & 173 & $1,3(1,0-1,8)$ & $0,8(0,5-1,4)$ & $1,1(0,8-1,6)$ & $2,0(1,3-3,0)$ & $1,2(0,7-2,1)$ \\
\hline Método anticoncepcional & & $p<0,0001$ & $p=0,010$ & $p=0,030$ & $p<0,0001$ & $p=0,070$ \\
\hline Nenhum & 357 & 1,0 & 1,0 & 1,0 & 1,0 & 1,0 \\
\hline Pílula & 505 & $0,5(0,3-0,6)$ & $1,8(1,2-2,7)$ & $0,7(0,5-0,9)$ & $0,5(0,3-0,7)$ & $0,6(0,4-0,9)$ \\
\hline Hormonal injetável & 29 & $0,5(0,2-1,2)$ & $1,8(0,7-4,5)$ & $1,4(0,9-2,3)$ & $0,5(0,1-2,0)$ & $1,6(0,7-3,8)$ \\
\hline DIU & 26 & $0,7(0,3-1,3)$ & - & $0,6(0,2-1,6)$ & $1,2(0,6-2,5)$ & $0,7(0,2-3,2)$ \\
\hline Ligadura tubária & 96 & $1,2(0,9-1,7)$ & $2,1(1,2-3,6)$ & $1,1(0,7-1,8)$ & $0,7(0,4-1,1)$ & $1,2(0,6-2,1)$ \\
\hline Tabagismo & & $p=1,000$ & $p=0,800$ & $p=0,600$ & $p=0,300$ & $p=0,200$ \\
\hline Não & 750 & 1,0 & 1,0 & 1,0 & 1,0 & 1,0 \\
\hline $\operatorname{Sim}$ & 196 & $1,2(0,9-1,6)$ & $1,2(0,7-2,0)$ & $1,1(0,7-1,6)$ & $1,1(0,7-1,6)$ & $1,4(0,9-2,3)$ \\
\hline Ex-fumante & 136 & $0,9(0,6-1,4)$ & $1,1(0,6-1,8)$ & $0,9(0,6-1,3)$ & $1,4(0,9-2,1)$ & $0,9(0,4-1,8)$ \\
\hline
\end{tabular}

ABEP: Associação Brasileira de Empresas de Pesquisa; DIU: dispositivo intrauterino; IC95\%: intervalo de $95 \%$ de confiança; IMC: índice de massa corporal; RP: razão de prevalência.

* Teste Wald para tendência linear.

"ligadura ou nenhum método". Tais análises mostraram que, exceto para o sangramento intermenstrual, as prevalências de todos os distúrbios menstruais foram maiores entre as mulheres que tinham ligadura ou não usavam nenhum método anticoncepcional. Da mesma forma, após reca- tegorizar a variável "método anticoncepcional" em "uso de pílula, injeção ou DIU", "ligadura" e "nenhum método", as maiores frequências dos distúrbios foram todas entre aquelas que faziam uso de ligadura. 


\section{Discussão}

Em resumo o presente estudo mostrou que os distúrbios menstruais são frequentes na população feminina de 15-54 anos de idade e associam-se à paridade, IMC e tipo de método anticoncepcional. A paridade se associou com distúrbios menstruais de intensidade (sangramento excessivo), duração (menstruação prolongada) e periodicidade (ciclos curtos). O IMC se associou com distúrbios da intensidade (sangramento excessivo) e da periodicidade (ciclo longo). Já o método anticoncepcional influiu nos distúrbios de intensidade e periodicidade.

Estudos de base populacional sobre distúrbios menstruais são escassos na literatura. A maioria dos trabalhos relacionados ao tema foi realizada em clínicas especializadas em saúde da mulher ou em postos de atendimento médico, que incluem uma população específica, impedindo que os dados sejam extrapolados para a população geral. Poucos estudos avaliam a ocorrência de um distúrbio menstrual isolado. Quando isso ocorre, a maioria descreve a ocorrência de sangramento menstrual excessivo 21,23,24,25,26, possivelmente, por esse ser o distúrbio mais frequente ou por sua presença ser mais incômoda para a mulher do que os demais distúrbios. Até onde sabem os autores, este é o primeiro estudo de base populacional realizado no país com o objetivo de investigar a prevalência de diferentes distúrbios menstruais entre mulheres de 15-54 anos de idade.

Quase metade da população apresentou pelo menos um sintoma menstrual no período de seis meses (46,4\%; IC95\%: 43,3-49,4), sendo tal prevalência superior à encontrada na literatura. Dados de duas revisões sistemáticas apontam uma prevalência em torno de 5 a $30 \%$ para distúrbios menstruais entre mulheres de outros países 27,28 . Essa larga amplitude pode ser explicada pelo fato de as revisões sistemáticas incluírem populações com características de idade, nível econômico e escolaridade diferentes, além de variações entre os estudos, tanto na definição dos desfechos, quanto na interpretação das perguntas por parte das entrevistadas.

No grupo de mulheres que relataram presença de dois distúrbios nos últimos seis meses, as alterações do intervalo entre uma menstruação e outra (ciclo longo e ciclo curto) foram as mais frequentes. Esse resultado é consistente com os achados de um estudo com mulheres de 18-25 anos de idade, em Taiwan, que relatou que, para mais de um terço das entrevistadas, havia uma variação para mais ou para menos de sete dias na duração do ciclo menstrual 29 .

\section{Sangramento menstrual excessivo}

As prevalências de sangramento menstrual excessivo encontradas na literatura variam muito, com estudos mencionando frequências de $4 \% 27$ até $52 \% 30$. O atual estudo encontrou uma prevalência inferior à metade da encontrada em uma pesquisa que entrevistou mulheres entre 18 e 54 anos de idade, usuárias de um serviço de saúde em Londres, Inglaterra (52\%) 30 . Por outro lado, a prevalência observada no presente estudo foi quase três vezes maior que a encontrada em um estudo na Síria (8\%), conforme relatado em uma revisão de literatura 27 .

Variáveis demográficas, socioeconômicas e comportamentais estiveram associadas com a presença de sangramento menstrual excessivo. Mulheres com idade entre 35 e 44 anos apresentaram maiores prevalências desse distúrbio, sendo tais achados condizentes com a literatura 30 . No atual estudo, a menor escolaridade (0-4 anos), maior paridade ( $\geq 4$ partos), IMC $\geq 30,0$ e a ligadura tubária estiveram associadas com aumento na prevalência de sangramento menstrual excessivo. Corroborando esses achados, uma pesquisa realizada entre mulheres atendidas em postos de saúde na cidade de Pelotas 21, demonstrou que mulheres com menor nível de escolaridade (0-4 anos), maior paridade ( $\geq 4$ partos) e com ligadura tubária apresentaram maiores frequências de sangramento menstrual excessivo, sendo a prevalência entre essas de 40,2\%, 51,5\% e 68,5\%, respectivamente.

A associação observada entre tabagismo e sangramento excessivo foi também verificada entre adolescentes de 12-19 anos na Malásia 31 e entre mulheres de 18-49 anos de idade na Inglaterra 32 . Outro estudo realizado em Boston, Estados Unidos, com mulheres de 36-44 anos, encontrou associação entre sangramento menstrual excessivo e maior paridade 33 .

\section{Sangramento intermenstrual}

A prevalência encontrada no atual estudo $(11,1 \%)$ é compatível com os achados de uma revisão sistemática realizada por Harlow \& Campbell, em $2004{ }^{27}$, que mostrou que a prevalência de sangramento intermenstrual variou de $5 \%$ a $17 \%$. O sangramento intermenstrual associou-se apenas com o tipo de método anticoncepcional utilizado, e a maior frequência foi observada entre aquelas que apresentavam ligadura tubária. Não foram localizados na literatura artigos que tenham analisado a prevalência desse distúrbio conforme variáveis demográficas, socioeconômicas, comportamentais ou biológicas. 


\section{Ciclo menstrual curto}

A frequência de ciclo menstrual curto $(19,2 \%)$ foi semelhante à observada em um estudo realizado em 2007, na Etiópia, com adolescentes de 12-19 anos, que encontrou uma prevalência de $20,7 \% 34$. Contudo, dados apresentados em uma revisão sistemática apontam prevalências em torno de $1 \%$ a $6 \%$ em países em desenvolvimento 27 . Estudo conduzido na Nigéria mostrou uma prevalência de 12,1\% para adolescentes de 10-19 anos 35. Na Inglaterra, em 2004, a maior prevalência desse distúrbio foi relatada por $34,3 \%$ das mulheres com mais de 45 anos 30 .

\section{Ciclo menstrual longo}

Pesquisa realizada em Londres 36 , com mulheres entre 18-39 anos de idade, e estudo realizado na Etiópia ${ }^{34}$, somente com adolescentes, encontraram prevalências menores desse distúrbio (respectivamente, $4,8 \%$ e $9 \%$ ) do que a verificada em Pelotas (14,8\%). Resultados de uma revisão sistemática apontam prevalências que variam de 8 a $22 \% 27$. No presente estudo, esse distúrbio esteve associado com a idade (45-54 anos), IMC $\geq 30,0$ e uso de DIU. Estudo realizado na Inglaterra, em 2004 30, mostrou maiores prevalências de ciclo menstrual longo na mesma faixa etária. Outro estudo conduzido entre enfermeiras taiwanesas, com idades entre 25 e 35 anos, encontrou prevalência de $13,7 \% 37$.

\section{Menstruação prolongada}

Resultado superior à prevalência observada em Pelotas $(7,9 \%)$ foi relatado no estudo supracitado com enfermeiras taiwanesas $37(33,8 \%)$. Por outro lado, dados de uma pesquisa realizada na Nigéria, somente com adolescentes, encontrou uma prevalência menor (3,6\%) 35 . Consistente com os achados do atual estudo, os dados da pesquisa realizada por Abenhaim \& Harlow 33 mostraram que mulheres que tiveram mais de três partos relataram a ocorrência de menstruação prolongada mais frequentemente do que as nulíparas.

A associação verificada entre tabagismo e menstruação prolongada é consistente com os achados do estudo na Inglaterra, em 1988, com mulheres de 18-49 anos de idade, que encontrou uma prevalência de $14,5 \%$ entre tabagistas 32 .

\section{Limitações do estudo}

Este estudo apresenta algumas limitações. Primeiro, pelo fato de estar inserido em um consórcio de pesquisa, houve restrição no número de questões abordadas, limitando a coleta de outras informações relacionadas aos distúrbios menstruais. Aspectos como a investigação detalhada da história reprodutiva da mulher (por exemplo, idade da menarca 31,33,38, tempo de uso do atual método anticoncepcional ${ }^{39}$ ), presença de sintomas menstruais (como dismenorreia) 31,34 e ginecológicos (dispareunia) ${ }^{40}$, histórico de consultas médicas (com médicos ginecologistas ou não) 33,34 e presença de fatores estressantes 38 , cuja associação foi investigada por outros autores, não puderam ser explorados.

Segundo, todas as informações foram referidas pela própria mulher, podendo ter ocorrido erros na definição dos distúrbios menstruais. Diferentemente da duração e da periodicidade, que são atributos quantitativos da menstruação e do ciclo menstrual, a intensidade tem um componente subjetivo, dependendo da percepção da própria mulher. Há evidências de que a percepção do sangramento menstrual excessivo, por exemplo, depende da experiência menstrual prévia da mulher e de aspectos econômicos e culturais, que incluem o uso habitual ou não de absorventes higiênicos durante a menstruação e o tipo de absorventes utilizados 41 . Entretanto, a avaliação objetiva da quantidade de perda sanguínea menstrual, como a utilização do método da hematina alcalina 42 , é impraticável, tanto na clínica 43 , quanto em estudos epidemiológicos.

Como o número de mulheres na faixa etária de interesse foi inferior ao estimado no cálculo do tamanho amostral, o número final na amostra (1.078 mulheres) correspondeu a cerca da metade do previsto. Com esse tamanho de amostra, a precisão das estimativas de prevalência caiu de três para pouco mais de quatro pontos percentuais. Por outro lado, o cálculo a posteriori dos DEF para cada distúrbio menstrual encontrou valores iguais ou inferiores a 1,2, diminuindo a correlação intraclasse e otimizando o tamanho real da amostra.

Finalmente, deve-se destacar que a maior prevalência desses distúrbios menstruais pode ser devida a outros fatores: (a) ao caráter de cronicidade descrito para esses sintomas 44,45 , aspecto que o atual estudo não investigou; (b) a não percepção de desconforto por parte da mulher; (c) ao fato de não considerarem como "distúrbio” esses desconfortos, por conta da visão social que naturaliza aquilo que se costuma chamar de "problemas femininos" 46; ou (d) por maior dificuldade de acesso a serviços especializados de saúde. Estudos futuros poderão ajudar a esclarecer as razões dessa diferença e, inclusive, explicar a plausibilidade biológica e social para os atuais achados.

Apesar das limitações, esse estudo contribui para a literatura científica por apresentar as 
prevalências de vários distúrbios menstruais, obtidos de uma amostra de base populacional. Tais achados poderão auxiliar no planejamento de ações e serviços de saúde, especialmente os de atenção básica, que são a porta de entrada do maior contingente de mulheres que referiram a presença de distúrbios menstruais (menos escolarizadas, com maior paridade e fumantes) 21,27.

\section{Resumen}

Con el fin de determinar la prevalencia de los trastornos menstruales y su distribución, de acuerdo a la paridad y a variables demográficas, sociales y de comportamiento, entre los 15-54 años, se realizó un estudio transversal, basado en la población de Pelotas, Rio Grande do Sul, Brasil, con 1.078 mujeres. Se investigaron el sangrado menstrual excesivo, sangrado, el ciclo menstrual, ciclo menstrual corto y el periodo de menstruación prolongada. Se calcularon las tasas de prevalencia e incidencia, con intervalos de confianza del 95\%. La prevalencia global de los trastornos menstruales fue de un 46,4\% $(43,4-49,4)$. El sangrado menstrual excesivo fue el más prevalente (23,2\%; 20,5-25,8), seguido por ciclo menstrual corto $(19,2 \% ; 16,6-21,8)$, durante los ciclos menstruales (14,8\%; 12,7-16,9), hemorragia $(11,1 \% ; 9,1-13,2)$ y prolongada menstruación $(7,9 \%$; 6,3-9,4). Los trastornos menstruales son frecuentes en la población, y se asocian con la paridad, índice de masa corporal y método anticonceptivo.

Trastornos de la Menstruación; Menstruación; Salud de la Mujer

\section{Colaboradores}

R. S. Barcelos participou de todas as etapas do trabalho de campo e foi responsável pela revisão da literatura, análise dos dados e redação do artigo. R. V. Zanini colaborou na revisão e análise dos dados e do artigo. I. S. Santos orientou todas as etapas da pesquisa e revisou o artigo.

\section{Agradecimentos}

Nossos agradecimentos à Capes pelo apoio financeiro. 


\section{Referências}

1. Treloar AE, Boynton RE, Behn BG, Brown BW. Variation of the human menstrual cycle through reproductive life. Int J Fertil 1967; 12:77-126.

2. Thomas F, Renaud F, Benefice E, de Meeus T, Guegan JF. International variability of ages at menarche and menopause: patterns and main determinants. Hum Biol 2001; 73:271-90.

3. Cutright P. The teenage sexual revolution and the myth of an abstinent past. Fam Plann Perspect 1972; 4:24-31.

4. Al-Sahab B, Ardern CI, Hamadeh MJ, Tamim H. Age at menarche in Canada: results from the National Longitudinal Survey of Children \& Youth. BMC Public Health 2010; 10:736.

5. Benjamin F. The age of the menarche and of the menopause in white South African women and certain factors influencing these times. S Afr Med J 1960; 16:316-20.

6. Pedro AO, Pinto Neto AM, Paiva LHSC, Osis MJ, Hardy E. Idade de ocorrência da menopausa natural em mulheres brasileiras: resultados de um inquérito populacional domiciliar. Cad Saúde Pública 2003; 19:17-25.

7. Abramson JH, Gampel B, Slome C, Scothc N, Majola CC. Age at menopause of urban Zulu women. Science 1960; 132:356-7.

8. Wilbush J. Menorrhagia and menopause: a historical review. Maturitas 1988; 10:5-26.

9. Chiazze Jr. L, Brayer FT, Macisco Jr. JJ, Parker MP, Duffy BJ. The length and variability of the human menstrual cycle. JAMA 1968; 203:377-80.

10. Harlow SD, Ephross SA. Epidemiology of menstruation and its relevance to women's health. Epidemiol Rev 1995; 17:265-86.

11. Freitas F, Menke CH, Rivoire W, Passos EP. Rotinas em ginecologia. Porto Alegre: Editora Artmed; 2003.

12. Enciso FB, Crespo LP, Acuña JC. Classification and nomenclature of menstrual alterations. Ginecol Obstet Mex 2007; 75:641-51.

13. Machado LV. Sangramento uterino disfuncional. Arq Bras Endocrinol Metab 2001; 45:375-82.

14. Batista Filho M, Rissin A. Transição nutricional no Brasil: tendências regionais e temporais. Cad Saúde Pública 2003; 19:S181-91.

15. Martínez J, Araújo C, Horta BL, Gigante DP. Padrões de crescimento na infância precoce e ocorrência de menarca antes de doze anos de idade. Rev Saúde Pública 2010; 44:249-60.

16. Chandra A, Martinez GM, Mosher WD, Abma JC, Jones J. Fertility, family planning, and reproductive health of U.S. women: data from the 2002 National Survey of Family Growth. Vital Health Stat 2005; 25:1-160.

17. Santos IS, Barros AJD, Matijasevich A, Tomasi E, Medeiros RS, Domingues MR, et al. Mothers and their pregnancies: a comparison of three population-based cohorts in Southern Brazil. Cad Saúde Pública 2008; 24 Suppl 3:S381-9.

18. Ministério do Planejamento, Orçamento e Gestão. Perfil das mulheres responsáveis pelos domicílios no Brasil. http://www.ibge.gov.br/home/estatisti ca/populacao/perfildamulher/perfilmulheres.pdf (acessado em 21/Jun/2011).
19. Araújo OD, Cunha AL, Lustosa LR, Nery IS, Mendonça RCM, Campelo SMA. Aleitamento materno: fatores que levam ao desmame precoce. Rev Bras Enferm 2008; 61:488-92.

20. Barros AJD, Menezes AMB, Santos IS, Assunção MC, Gigante D, Fassa AG, et al. O Mestrado do Programa de Pós-graduação em Epidemiologia da UFPel baseado em consórcio de pesquisa: uma experiência inovadora. Rev Bras Epidemiol 2008; 11:133-44.

21. Santos IS, Minten GC, Valle NCJ, Tuerlinckx GC, Silva AB, Pereira GAR, et al. Menstrual bleeding patterns: a community-based cross-sectional study among women aged 18-45 years in Southern Brazil. BMC Women's Health 2011; 11:26.

22. Hatcher R, Rinehart W, Blackburn R, Geller J, Shelton J. Pontos essenciais da tecnologia de anticoncepção: um manual para pessoal clínico. Baltimore: Universidade Johns Hopkins; 2001.

23. Warner PE, Critchley HOD, Lumsden MA, BrownCampbell M, Douglas A, Murray GD. Menorrhagia II: is the $80-\mathrm{mL}$ blood loss criterion useful in management of complaint of menorrhagia? Am J Obstet Gynecol 2004; 190:1224-9.

24. Warner PE, Critchley HOD, Lumsden MA, BrownCampbell M, Douglas A, Murray GD. Menorrhagia I: measured blood loss, clinical features, and outcome in women with heavy periods: a survey with follow-up data. Am J Obstet Gynecol 2004; 190:1216-23.

25. Janssen CAH, Scholten PC, Heintz PM. Menorrhagia: a search for epidemiological risk markers. Maturitas 1997; 28:19-25.

26. O'Flynn N, Britten N. Menorrhagia in general practice: disease or illness. Soc Sci Med 2000; 50:651-61.

27. Harlow SD, Campbell OM. Epidemiology of menstrual disorders in developing countries: a systematic review. BJOG 2004; 111:6-16.

28. Agudelo LEP. Hemorragía uterina anormal: enfoque basado en evidencias. Revisión sistemática. Rev Med 2007; 15:68-79.

29. Chang PJ, Chen PC, Hsieh CJ, Chiu LT. Risk factors on the menstrual cycle of healthy Taiwanese college nursing students. Aust N Z J Obstet Gynaecol 2009; 49:689-94.

30. Shapley M, Jordan K, Croft PR. An epidemiological survey of symptoms of menstrual loss in the community. Br J Gen Pract 2004; 54:359-63.

31. Lee LK, Chen PC, Lee KK, Kaur J. Menstruation among adolescent girls in Malaysia: a cross-sectional school survey. Singapore Med J 2006; 47: 869-74.

32. Brown S, Vessey M, Stratton I. The influence of method of contraception and cigarette smoking on menstrual patterns. Br J Obstet Gynaecol 1988; 95:905-10.

33. Abenhaim HA, Harlow BL. Live births, cesarean sections and the development of menstrual abnormalities. Int J Gynaecol Obstet 2006; 92:111-6.

34. Zegeye DT, Megabiaw B, Mulu A. Age at menarche and the menstrual pattern of secondary school adolescents in northwest Ethiopia. BMC Women's Health 2009; 9:29. 
35. Nwankwo TO, Aniebue UU, Aniebue PN. Menstrual disorders in adolescent school girls in Enugu, Nigeria. J Pediatr Adolesc Gynecol 2010; 23:358-63.

36. Anastasakis E, Kingman CE, Lee CA, Economides DL, Kadir RA. Menstrual problems in university students: an electronic mail survey. In Vivo 2008; 22:617-20.

37. Lin HT, Lin CT, Shiao JS. The impact of self-perceived job stress on menstrual patterns among Taiwanese nurses. Ind Health 2007; 45:709-14.

38. Harlow SD, Campbell B, Lin X, Raz J. Ethnic differences in the length of the menstrual cycle during the postmenarcheal period. Am J Epidemiol 1997; 146:572-80.

39. Datey S, Gaur LN, Saxena BN. Vaginal bleeding patterns of women using different contraceptive methods (implants, injectables, IUDs, oral pills): an Indian experience. An ICMR Task Force Study. Indian Council of Medical Research. Contraception 1995; 51:155-65.

40. Mahmood TA, Templeton AA, Thomson L, Fraser C. Menstrual symptoms in women with pelvic endometriosis. Br J Obstet Gynaecol 1991; 98:558-63.
41. Santer M, Wyke S, Warner P. What aspects of periods are most bothersome for women reporting heavy menstrual bleeding? Community survey and qualitative study. BMC Womens Health 2007; 7:8.

42. Hallberg L, Nilsson L. Determination of menstrual blood loss. Scand J Clin Lab Invest 1964; 16:244-8.

43. Fernández JG. Hemorragia uterina disfuncional: diagnóstico y tratamiento. http://www.hvn.es/ servicios_asistenciales/ginecologia_y_obstetricia/ ficheros/cr08.hemorragia_uterina_disfuncional. pdf (acessado em 07/Jul/2011).

44. Berek J. Tratado de ginecologia. Rio de Janeiro: Editora Guanabara-Koogan; 2010.

45. Bankowski B, Hearne A, Lambrai N, Fox H, Walloch E. Manual de ginecologia e obstetrícia do Johns Hopkins. Baltimore: Department of Gynecology and Obstetrics, Johns Hopkins University School of Medicine; 2006.

46. Ferreira P, Barbosa E. Avaliação da percepção dos desconfortos menstruais entre mulheres que frequentam uma unidade de ensino e saúde. Revista de Pesquisa em Saúde 2012; 1:117-30.

Recebido em 08/Jan/2013

Versão final reapresentada em 29/Abr/2013

Aprovado em 15/Mai/2013 